\title{
Generation of broadband single-photon states in photonic-crystal fibers with flattened dispersion profiles
}

\author{
Ilya Chuprina ${ }^{2}$, and Ilnur Latypov ${ }^{1,2, *}$ \\ ${ }^{1}$ Kazan Quantum Center Kazan National Research Technical University after A.N. Tupolev-KAI, \\ 18a Chetaeva str., Kazan, 420126 Russia \\ ${ }^{2}$ Kazan E. K. Zavoisky Physical-Technical Institute, 10/7 Sibirsky tract Str., Kazan, 420029 Russia
}

\begin{abstract}
We theoretically analyze the capability of generating broadband single-photon states in photonic crystal fibers (PCF) formed by spontaneous four-wave mixing (SFWM) using femtosecond laser pulses. The design of PCF structures with the flat dispersion profile is proposed. The characteristics of the correlated photon pairs with a broadband spectrum and low spectral correlation for these structures are calculated.
\end{abstract}

\section{Introduction}

Spontaneous four-wave mixing (SFWM) and spontaneous parametric down-conversion (SPDC) is a powerful tool for generating a wide class of non-classical states, in particular the single-photon or two-photon states $[1,2]$. Such states are the basis for long-distance quantum communication, quantum memory and quantum computing protocols. Specific characteristics of such sources determine possibility of its application [3]. The main advantages of these methods include high stability of obtained states in time, the possibility to generate spectrally limited pulses at room temperatures, the possibility to adjust optical wavelength and the spectrum width as well as small influence of multi-photon states. The basic disadvantages of the methods are the random nature of single-photon generation, that leads to a probability of obtaining the two-photon state, and a correlation between the signal and idler photons. An important characteristic of single-photon pulse is the width of the spectrum. It determines the efficiency of interaction with the device quantum information science. An important characteristic of a single-photon pulse is the spectrum line width. It determines the efficiency of interaction with the quantum information device. For example, for cold atomic systems it is required to have a narrowband single-photon state with a spectral line width about $100 \mathrm{MHz}$. However, for some problems of interest one would need the spectral limited broadband single-photon pulses [4]. Broadband single-photon sources based on spontaneous four-wave mixing with fiber structures are of interest for high-speed transmission of information and high agreement with fiber networks. In this paper we investigate the possibility of generation such states using photonic-crystal fibers. Specific

Corresponding author: bibidey@mail.ru 
design structures of such fibers allow us to obtain a flat profile of dispersion at wavelengths of pump, signal and idler photons.

\section{Results}

In order to determine spectrum and correlation characteristics for the SFWM photon fields it's necessary to determine the exact values of the refraction index and dispersion of PCFs at its length, that take part in the parametric processes. Usually it is not possible to get analytical solution for the complex internal structure of PCFs to determine its characteristics and to find fundamental modes. That's why computations of the effective refraction index, dispersion and other characteristics of PCF are provided by means of a numerical solution of Maxwell's equations. In the study, simulations were carried out in Lumerical software.

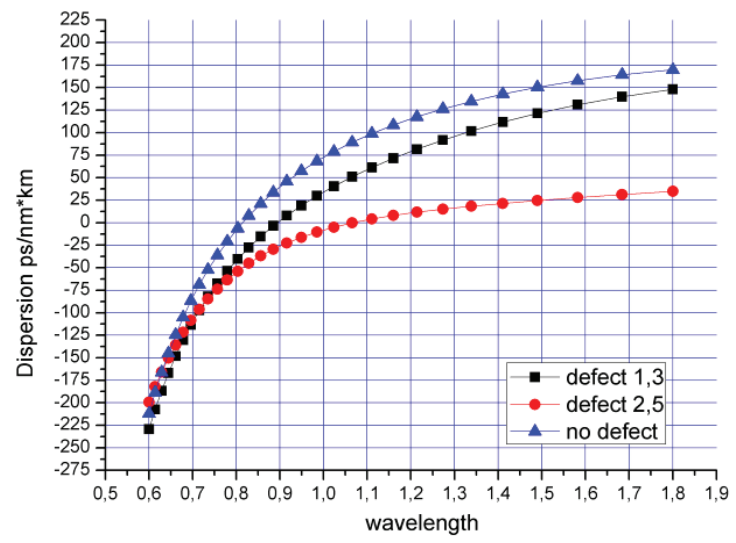

Fig. 1. . The dependence of the fiber dispersion for different structures on the wavelength. Fiber with "no defect" have zero dispersion wavelength at $800 \mathrm{~nm}$. Fiber with "defect 1,3" and "defect 2,5" have circle air hole in core with diameter 260 and $500 \mathrm{~nm}$.

We proposed a new design of photonic crystal fiber structure with the flattened dispersion profile optimized for generation of broadband single photon states in SFWM process using ultrashort laser pulses. Calculation of synchronism conditions showed a significant broadening of the spectrum compared to the standard photonic crystal fiber with low spectral correlation between the signal and idler photon.

The reported study was funded by RFBR according to the research projects № 16-02-00972.

\section{References}

[1] S.A. Castelletto, R.E. Scholten, Eur. Phys. J. 41(3), 181 (2008)

[2] C.J. Chunnilall et al., Opt. Engineering 53(8), 081910 (2014)

[3] A. Migdall, S.V. Polyakov, J. Fan, J.C. Bienfang, Single-Photon Generation and detection (Elsevier, Oxford, 2013)

[4] M.R. Sprague, P.S. Michelberger, T.F.M. Champion, D.G. England, J. Nunn, X.-M. Jin, W.S. Kolthammer, A. Abdolvand, P.St.J. Russell, I.A. Walmsley, Nature Photon. 8, 287 (2014) 